\title{
THE CLASSIFYING SPACE OF A PERMUTATION REPRESENTATION(')
}

\author{
BY
}

JAMES V. BLOWERS

\begin{abstract}
In this article the concept of classifying space of a group is generalized to a classifying space of an arbitrary permutation representation. An example of this classifying space is given by a generalization of the infinite join construction that defines the standard example of a classifying space of a group. In a previous paper of the author, the join of two permutation representations was defined, and it was shown that the cohomology ring of the join was trivial. In this paper the classifying space of the join of two permutation representations is shown to be the topological join of the two classifying spaces and from this the triviality of the cup-product is derived topologically.
\end{abstract}

I. Introduction. In this article the concept of classifying space of a group is generalized to a classifying space of an arbitrary permutation representation. It will be shown that the cohomology groups of this classifying space (with coefficients in $Z$ ) are equal to the corresponding cohomology groups of the permutation representation. (The cohomology theory of permutation representations can be found in Snapper [9] and Harris [6].) The standard example of a classifying space of a group will be generalized to a classifying space of a permutation representation.

In a previous paper of the author [1], it was proved that the cohomology of the join of two permutation representations (to be defined later) has trivial cup-products. That paper noted that the algebraic, computational proof of this result was lengthy. In this paper it will be shown that a classifying space for the join of two permutation representations is given by the topological join of their classifying spaces, which will lead to a brief topological proof of this result.

Received by the editors November 18, 1975.

AMS (MOS) subject classifications (1970). Primary 55F35, 55F40; Secondary 18H10, $20 \mathrm{~B} 05$.

Key words and phrases. Classifying space, permutation representation, join, cell complexes, cohomology ring, cup-products, equivariantly acyclic carriers, equivariantly proper maps, contractible spaces.

(I) This paper is based on a dissertation presented to the faculty of Northwestern University in partial fulfillment of the requirements of the degree of Doctor of Philosophy, June, 1972. The work was supported in part by National Defense Education Act Title IV Fellowship No. 68-01201. 
II. Preliminaries. We will work in the category of regular cell complexes, using much of the terminology of Cooke and Finney [4]. In particular, the following result will be used:

LEMmA 2.1. Let the group $G$ act cellularly on a cell-complex $E$. Then the incidence relation on $E$ can be defined in such a way that the action of $G$ commutes with the boundary operator.

Proof. Straightforward.

Let $(G, X)$ be a permutation representation and let $x \in X$. The family of subgroups $\mathfrak{G}=\left\{H_{x} \mid x \in X\right\}$ is called the set of stabilizers of $(G, X)$, and one says that $G$ acts on $X$ with stabilizers in $\mathscr{G}$ if $\mathscr{G} \supset\left\{H_{x} \mid x \in X\right\}$. If $G$ acts cellularly on a cell-complex $E$, then the stabilizers $\mathfrak{G}$ of $(G, E)$ are defined to be the set

$$
\left\{H_{e} \mid e \text { a point of } E\right\}=\left\{H_{e} \mid e \text { a cell of } E\right\} .
$$

A space $E$ is $H$-contractible if there is a contracting homotopy which commutes with elements of $H$. If for all $H \in \mathfrak{G}$, there is an $H$-homotopy from the identity map of $E$ to the constant map on $v$, where $v \in E$ depends on $H$, then one says that $E$ is $\mathfrak{g}$-contractible.

A similar terminology is used for $(Z) G$-chain complexes. Let $C$ be such a complex. Then $C$ is $H$-acyclic if there is an $H$-chain homotopy $s: C \rightarrow C$ of degree 1 and an $H$-map $\mu: Z \rightarrow C_{0}$ such that $(\partial s+s \partial) c=c$ if $\operatorname{dim} c>0$, and $(\partial s+s \partial) c=c-\mu \in c$ if $\operatorname{dim} c=0$. The term $\mathfrak{S}$-acyclic, where $\mathfrak{G}$ is a family of subgroups of $G$, is defined in the obvious manner. Following Hochschild [7] and Harris [6], we define a $G$-module $P$ to be $\mathfrak{S}$-projective if for every $H$-acyclic sequence of $G$-modules $(H \in \mathfrak{S})$

$$
0 \rightarrow A \stackrel{f}{\rightarrow} B \stackrel{g}{\rightarrow} C \rightarrow 0
$$

and every $G$-map $h: P \rightarrow C$, there is a $G$-map $j: P \rightarrow B$ such that $g j=h$. If $\mathfrak{G}=\{H\}$, then the term $[G: H]$-projective will be used. (For more details on relative projectives, the reader is referred to Hochschild [7], Harris [6], and Eilenberg-Moore [5].)

Let $E$ and $E^{\prime}$ be cell complexes upon which the group $G$ acts, with stabilizers in $\mathfrak{Q}$ and $\mathfrak{Q}^{\prime}$ respectively, and let $C$ be a $G$-equivariant carrier from $E^{\prime}$ to $E$. Then $C$ is an equivariantly acyclic carrier if for any cell $\sigma$ of $E$, the complex $C(\sigma)$ is $H(\sigma)$-acyclic, where $H(\sigma)=\{h \in G \mid h \sigma=\sigma\}$. A map $f: E \rightarrow E^{\prime}$ is equivariantly proper if the minimal carrier for $f$ is equivariantly acyclic.

III. The classifying space. The following lemma is a generalization of Cooke and Finney [4, Lemma 2.4, p. 144]: 
LEMMA 3.1. Let $E$ and $E^{\prime}$ be cell complexes upon which $G$ operates, and suppose it operates with stabilizers in $\mathfrak{B}$ on $E$ and $\mathfrak{S}^{\prime}$ on $E^{\prime}$, where $\mathfrak{S} \subset \mathfrak{B}^{\prime}$. Let $C$ be an equivariantly acyclic carrier from $E$ to $E^{\prime}$. Then $C$ carries a chain map $\varphi: C_{*}(E) \rightarrow C_{*}\left(E^{\prime}\right)$ which is a $G$-homomorphism of augmented complexes, such that for all cells $\sigma$ of $E$, the chain $\varphi \sigma$ is a chain of $C(\sigma)$. Further, if $\varphi_{0}$ and $\varphi_{1}$ are two such chain maps, then $\varphi_{0}$ and $\varphi_{1}$ are related by a G-chain homotopy $D$ carried by $C$.

Proof. The proof is by induction on dimension. Select a representative from each of the $G$-orbits of $n$-cells of $E$ (of $E^{\prime}$ ) and call the set of representatives $T_{n}\left(T_{n}^{\prime}\right)$. For every vertex $v \in T_{0}, C(v)$ is $H(v)$-acyclic; hence there is a vertex $v^{\prime} \in E^{\prime}$ such that $H(v)$ fixes $v^{\prime}$ and commutes with the augmentations. Set $\varphi(v)=v^{\prime}$. Extend $\varphi$ to all of $C_{0}(E)$ by action of $G$ and linearity.

Let $\sigma$ be a 1-cell of $T_{1}$. Then $\varphi \partial \sigma$ is a 0 -chain on $C(\sigma)$, fixed by $H(\sigma)$. Since $C(\sigma)$ is $H(\sigma)$-acyclic, there is an $H(\sigma)$-contracting homotopy $s$ on $C(\sigma)$ such that

$$
\partial s \varphi \partial \sigma=\varphi \partial \sigma-s \partial \varphi \partial \sigma=\varphi \partial \sigma .
$$

Set $\varphi \sigma=s \varphi \partial \sigma$, and extend to $C_{1}(E)$ by action of $G$ and linearity; $\varphi$ is well defined because $H(\sigma)$ fixes $\sigma$, hence fixes $s \varphi \partial \sigma$. This defines $\varphi$ in dimension 1 .

Suppose $\varphi$ has been defined on $C_{q}(E)$ for all $q<n$ so that it commutes with the boundary operators and with $G$ and is carried by $C$. Let $\sigma \in T_{n}$. Then $\varphi \partial \sigma$ is an $(n-1)$-chain on $C(\sigma)$. Let $s$ be an $H(\sigma)$ chain homotopy on $C(\sigma)$. Then

$$
\partial s \varphi \partial \sigma=\varphi \partial \sigma-s \partial \varphi \partial \sigma=\varphi \partial \sigma .
$$

Let $\varphi \sigma=s \varphi \partial \sigma$. Then $\partial \varphi \sigma=\varphi \partial \sigma$ and $H(\sigma) \varphi(\sigma)=\varphi(\sigma)$. Extend over all of $C_{n}(E)$ by linearity and action of $G$. This completes the construction of the chain map $\varphi: C_{*}(E) \rightarrow C_{*}\left(E^{\prime}\right)$.

The construction of the chain homotopy $D$ relating $\varphi_{0}$ to $\varphi_{1}$ is a straightforward extension of the above construction and the one in Cooke and Finney [4, p. 145].

COROLlaRY 3.1. If $f$ is an equivariantly proper map from $E$ to $E^{\prime}$ where $G$ acts with stabilizers in $\mathfrak{B}$ on $E$ and in $\mathfrak{Q}^{\prime}$ on $E^{\prime}$, where $\mathfrak{G} \subset \mathfrak{S}^{\prime}$, and $C_{0}$ and $C_{1}$ are two equivariantly acyclic carriers for $F$ (i.e., fo $\subset C_{0}(\sigma)$ and fo $\subset C_{1}(\sigma)$ for all cells $\sigma$ of $E$ ), then any two associated chain maps $\varphi_{0}$ for $C_{0}$ and $\varphi_{1}$ for $C_{1}$ are $G$ homotopic.

Proposition 3.1. Let $G$ operate with stabilizers in $\mathfrak{g}$ on $E$ and $E^{\prime}$, and let $f, g: E \rightarrow E^{\prime}$ be equivariantly proper maps of $G$-cell complexes, and let $H \in \mathfrak{W}$. If $F$ is an equivariantly proper $H$-homotopy from $f$ to $g$, then $f^{\#}$ and $g^{\#}$ (the 
associated chain maps) are related by an $H$-chain homotopy s.

Proof. Let $p_{0}$ and $p_{1}: E \rightarrow E \times I$ be defined by $p_{0}(e)=(e, 0)$ and $p_{1}(e)$ $=(e, 1)$. Then $p_{0}$ and $p_{1}$ are cellular and are equivariantly proper. In fact, the carriers are given by $C_{0}(\sigma)=\mathrm{Cl}(\sigma) \times v_{0}$, and $C_{1}(\sigma)=\mathrm{Cl}(\sigma) \times v_{1}$; both $C_{0}$ and $C_{1}$ are equivariantly acyclic. Define $\varphi_{0}, \varphi_{1}: C_{*}(E) \rightarrow C_{*}(E \times I)$ by $\varphi_{0}(\sigma)=\sigma \otimes v_{0}$ and $\varphi_{1}(\sigma)=\sigma \otimes v_{1}$, for $\sigma$ a cell of $E$. Then $C_{0}$ carries $\varphi_{0}$ and $C_{1}$ carries $\varphi_{1}$. Further, $\varphi_{0}$ is $H$-homotopic to $\varphi_{1}$, where the homotopy is defined by

$$
s(\sigma)=(-1)^{\operatorname{dim} \sigma} \sigma \otimes I
$$

where $\sigma$ is a cell of $E$.

The map $F$ is an equivariantly proper $H$-map, with carrier $C$, say. By Lemma 3.1, it induces a chain map $F^{\sharp}$. Define $C_{2}$ and $C_{3}$ by $\bullet$

$$
C_{2}(\sigma)=C\left(\sigma \times \mathrm{Cl}\left(v_{0}\right)\right), \quad C_{3}(\sigma)=C\left(\sigma \times \mathrm{Cl}\left(v_{1}\right)\right) .
$$

Then $C_{2}$ is an equivariantly acyclic carrier for $F \circ p_{0}=f$ and $C_{3}$ is an equivariantly acyclic carrier for $F \circ p_{1}=g$. Further, $C_{2}$ carries $F^{\sharp} \circ \varphi_{0}$ and $C_{3}$ carries $F^{\#} \circ \varphi_{1}$. Hence associated chain maps for $f$ and $g$ are $F^{\#} \circ \varphi_{0}$ and $F^{\sharp} \circ \varphi_{1}$, which are homotopic by the homotopy $F^{\sharp} \circ s$, proving the proposition.

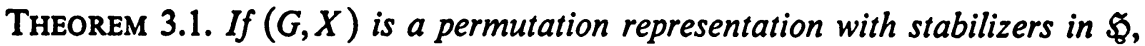

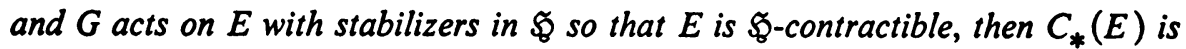
$H$-acyclic.

Proof. Select $H \in \mathfrak{B}$. Then there is an $H$-contracting homotopy $F_{H}$ : $E \times I \rightarrow E$ such that $F_{H}$ restricted to $E \times v_{0}$ is the projection on $E$ and $F_{H}$ restricted to $E \times v_{1}$ is the constant map on $v$. Now, by our previous convention we can choose $F_{H}$ to be equivariantly proper. We have $H v=v$. The identity map on $E$ and the constant map on $v$ are both cellular, inducing chain maps $i$, the identity on $C_{*}(E)$, and the chain map $\varphi$ respectively, where $\varphi(\sigma)=0$ unless $\operatorname{deg} \sigma=0$, whence $\varphi(\sigma)=v$. By Proposition 3.1, $i$ and $\varphi$ are related by an $H$-chain homotopy $s$, so that $\partial s+s \partial=i-\varphi$. Define $\mu(1)=v$. Then if $w$ is a 0 -cell of $E$, then $\partial s w+v=w$ or $\partial s w+\mu \varepsilon w=w$. Also $H \mu=\mu$. Thus $s$ is an $H$-contracting homotopy in dimension 0 . If $n \geqslant 1$, and $\sigma$ is of dimension $n$, then $\partial s \sigma+s \partial \sigma=i \sigma-\varphi \sigma=\sigma$. Hence $s$ is an $H$-contracting homotopy in

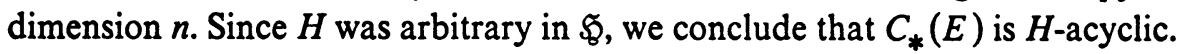

LEMMA 3.3. If $G$ acts on $E$ as above, then $C_{*}(E / G)=C_{*}(E) \otimes_{G} Z$.

TheOREM 3.2. Let $(G, X)$ and $\$$ be as in Theorem 3.1, and let $G$ act on an $\$-$ contractible space $E$ with stabilizers in $\mathfrak{B}$. Then if $A$ is a trivial $G$-module, then 


$$
H^{*}(E / G, A) \cong H^{*}((G, X), A)
$$

Proof. It is straightforward to show $C_{*}(E / G) \cong C_{*}(E) \otimes_{G} Z$. Then by Theorem 3.1,

$$
\begin{aligned}
H^{*}(E / G, A) & =H^{*}\left(\operatorname{Hom}\left(C_{*}(E / G), A\right)\right)=H^{*}\left(\operatorname{Hom}\left(C_{*}(E) \otimes_{G} Z, A\right)\right) \\
& =H^{*}\left(\operatorname{Hom}_{G}\left(C_{*}(E), \operatorname{Hom}(Z, A)\right)\right)=H^{*}\left(\operatorname{Hom}_{G}\left(C_{*}(E), A\right)\right) \\
& =H^{*}((G, X), A) .
\end{aligned}
$$

If $E$ is any cell complex that satisfies the hypotheses of Theorem 3.1, then we call $E / G$ a classifying space for $(G, X)$.

IV. The standard example. The standard example of a classifying space of a group $G$ is the space $J^{\infty} G$ modulo the action of $G$, where $J$ denotes the topological join. This generalizes readily to permutation representations. The notation for joins used here will be that of Cooke and Finney [4]. Specifically, Cooke and Finney define a raised chain complex of a cell complex $E$ to be the chain complex $s C$, where $s C_{q}(E)=C_{q-1}(E)$, if $q>0$, and $s C_{0}(E)=Z v_{E}$, where $\nu_{E}$ is a void cell of dimension -1 added to $E$. The differentials are given by $\partial_{q}^{\prime}=\partial_{q-1}$ if $q>0$, and $\partial_{0}=\varepsilon$, the augmentation of $C$. They then show that the chain complex of the join $E * E^{\prime}$ of two cell complexes $E$ and $E^{\prime}$ is given by:

$$
s C_{*}\left(E * E^{\prime}\right)=s C_{*}(E) \otimes s C_{*}\left(E^{\prime}\right)
$$

This construction generalizes readily to the join of $n$ cell complexes. Given a cell complex $E$, we define the cell complex $J^{\infty} E$ as the direct limit of the inductively ordered system:

$$
E=J^{1} E \subset J^{2} E \subset \cdots
$$

where $J^{n} E$ denotes the $n$-fold join of $E$ with itself. $J^{\infty} E$ is given the weak topology, which makes it into a regular cell complex.

Now let $(G, X)$ be a permutation representation with stabilizer set $\mathfrak{B}$. The set $X$ with the discrete topology is a regular complex of cells of dimension zero. Then $J^{\infty} X$ is a regular cell complex. A vertex of this complex will be denoted by $x(k)$, where $x \in X$, and $k \geqslant 0$ is an integer which tells in which of the factors of $J^{\infty} X$ the vertex is. A typical cell of $J^{\infty} X$ will be denoted by

$$
x_{0}\left(k_{0}\right) * x_{1}\left(k_{1}\right) * \cdots * x_{n}\left(k_{n}\right)=\stackrel{J}{J}=0_{i}^{n} x_{i}\left(k_{i}\right)
$$

where the $x_{i} \in X$ and $0 \leqslant k_{0}<k_{i}<\cdots<k_{n}$. (If an integer $k$ is not one of the $k_{i}$, then the corresponding factor of this cell is $\nu_{E}$.) The action of $G$ is 
defined componentwise on $J^{\infty} X$. Let $J=J^{\infty} X$. We shall prove that $J$ is $\$$ contractible and has the right stabilizers. To do this, we need this lemma:

LEMMA 4.1. Let $G$ act on a cell complex $E$ with stabilizers in $\mathfrak{S}$ and let $v \in E$ be a point fixed by $H \in \mathfrak{G}$. Then the cell complex $v * E$ is $H$-contractible and its chain complex is $\mathrm{H}$-acyclic.

Proof. The $H$-chain homotopy is given by the cellular map $F$ defined by

$$
F\left(\left(t_{0} v, t_{1} e\right), t\right)=\left(\left((1-t) t_{0}+t\right) v, t_{1}(1-t) e\right) .
$$

Proposition 4.1. Let $(G, X)$ be a permutation representation with stabilizers in $\mathfrak{B}$. Then for each $H \in \mathfrak{B}$ there is an H-homotopy $F: J \times I \rightarrow J$ that is equivariantly proper as a map of $H$-spaces such that $F$ defined on $J \times v_{0}$ is the projection of $J \times v_{0}$ onto $J$, and $F$ defined on $J \times v_{1}=$ constant map on a point $v$ fixed by $H$.

Proof. Regard $J$ as $J_{i=0}^{\infty} X$ and set $J^{\prime}=J_{i=1}^{\infty} X$. An injective simplicial map $m: J \rightarrow J$ is defined by

$$
m\left(x_{0}\left(k_{0}\right) * \cdots * x_{n}\left(k_{n}\right)\right)=x_{0}\left(k_{0}+1\right) * \cdots * x_{n}\left(k_{n}+1\right)
$$

if $0 \leqslant k_{0}<\cdots<k_{n}$, where $x_{i}\left(k_{i}\right)$ denotes $x_{i}$ in the $k_{i}$ th place. Then $m$ is an isomorphism from $J$ onto $J^{\prime}$. (The inverse map is obtained from the above by changing the plus signs to minus.) A map $F_{1}: J \times I \rightarrow J$ will now be defined which will be a $G$-homotopy from $i$ to $m$, where $i$ is the identity on $J \times I$. The vertex set of $J \times I$ is $J^{0} \times I^{0}$; i.e., the set of all ordered pairs $(x(k), \varepsilon)$, where $x(k)$ is a vertex of $J$ and $\varepsilon=0$ or 1 , which we shall identify with $v_{0}$ or $v_{1}$, respectively. A simplex of $J \times I$ is a collection of vertices $\left\{\left(x_{i}\left(k_{i}\right), \varepsilon_{i}\right)\right\}$ $i=0, \ldots, n\}$ such that for each $i=0, \ldots, n-1$, the inequalities $k_{i} \leqslant k_{i+1}$ and $\varepsilon_{i} \leqslant \varepsilon_{i+1}$ hold and strict inequality holds in at least one of these. Define the simplicial map $F_{1}^{\prime}$ by

$$
F_{1}^{\prime}(x(k), \varepsilon)=x(k+\varepsilon)
$$

A simplicial complex is a cell complex, so $F_{1}^{\prime}$ is a map of cell complexes. Further, $F_{1}^{\prime}$ is equivariantly proper, with the minimal acyclic carrier $C_{1}$ given by

$$
\begin{array}{r}
C_{1}\left(\sigma \times v_{0}\right)=C_{*}(\mathrm{Cl}(\sigma)), \quad \text { if } \sigma \in J, \\
C_{1}\left(\sigma \times v_{1}\right)=C_{*}(\mathrm{Cl}(m(\sigma))), \quad \text { if } \sigma \in J, \\
C_{1}\left(J x_{i}\left(k_{i}\right) \times I\right)=C_{*}\left(\cup \left(\mathrm { Cl } \left(x_{0}\left(k_{0}\right) * \cdots * x_{i}\left(k_{i}\right) * x_{i}\left(k_{i+1}\right)\right.\right.\right. \\
\left.\left.\left.* \cdots * x_{n}\left(k_{n+1}\right)\right)\right)\right),
\end{array}
$$


where $C_{*}(E)$ denotes the chain complex of $E$. It is also a $G$-homotopy, because the group action does not change the order of the factors.

By Lemma 4.1, if $v \in X$ such that $H v=v$, then $v(0) * J^{\prime}$ is $H$-contractible. Hence there is an equivariantly proper $H$-homotopy

$$
F_{2}^{\prime}:\left(v(0) * J^{\prime}\right) \times I \rightarrow v(0) * J^{\prime}
$$

from the identity on $v(0) * J^{\prime}$ to the constant map on $v(0)$. The inclusion $i: v(0) * J^{\prime} \rightarrow J$ composed with $F_{2}^{\prime}$ yields an equivariantly proper $H$-homotopy $F_{2}$ from $i$ to the constant map on $v(0)$.

Define $F: J \times I \rightarrow J$ by

$$
\begin{array}{ll}
F(x, t)=F_{1}(x, 2 t) & \text { if } 0 \leqslant t \leqslant \frac{1}{2}, \\
F(x, t)=F_{2}\left(m(x), 2\left(t-\frac{1}{2}\right)\right) & \text { if } \frac{1}{2} \leqslant t \leqslant 1 .
\end{array}
$$

Then $F$ is an equivariantly proper $H$-homotopy from the identity on $J$ to the constant map on $v(0)$, where the minimal carrier $C$ for $F$ is given by:

$$
C\left(\sigma \times v_{0}\right)=C_{1}\left(\sigma \times v_{0}\right)=C_{*}(\mathrm{Cl}(\sigma)), \quad C\left(\sigma \times v_{1}\right)=C_{*}(\mathrm{Cl}(v(0))),
$$

and $C(\sigma \times I)$ is the chain complex of the union of the cell complex whose associated chain complex is $C_{1}(\sigma)$, and the cell complex $\mathrm{Cl}(\nu(0) * m(\sigma))$. It can be checked that $C$ is equivariantly acyclic, showing $F$ to be equivariantly proper as an $H$-map. This proves the proposition.

COROllaRY 4.1. The cell complex $J$ is $\mathfrak{S}$-contractible, and $C_{*}(J)$ is $\mathfrak{Z}$-acyclic.

Proof. By Theorem 3.1.

Proposition 4.2. The chain complex $C_{*}(J)$ is $\mathfrak{S}$-projective.

Proof. Let $\sigma=v_{0} * \cdots * v_{n}$ be a cell of $J$. Then $g \sigma=\sigma$ if and only if $g \in H=\bigcap_{i=0}^{n} H_{i}$, where $H_{i}$ stabilizes $v_{i}$. Although $H$ may not be in $\mathfrak{Q}$, it will be if we replace $\mathfrak{B}$ by

$$
\mathfrak{S}^{\prime}=\left\{\bigcap_{i=0}^{n} H_{i} \mid H_{i} \in \mathfrak{G}\right\} .
$$

Both $\mathscr{B}$ and $\mathscr{G}^{\prime}$ lead to the same sets of projective objects and exact sequences (see Eilenberg-Moore [5, Chapter 2, Theorem 3.1]). Hence $C_{*}(J)$ is $\mathfrak{S}^{\prime}$ projective and thus $\mathfrak{B}$-projective.

By Theorem 3.2, we have $H^{*}(J / G, A)=H^{*}((G, X), A)$. This completes the description of the standard example of a classifying space for $(G, X)$. If $X=G$ and $(G, G)$ is the regular representation, then the classifying space is the standard example of the classifying space of a group. (See Milnor [8].) We 
shall denote a classifying space for $(G, X)$ by $B_{(G, X)}$.

As it turns out, the theory of classifying spaces of permutation representations is more complicated than the theory of classifying spaces of groups. Many theorems about classifying spaces of groups do not hold for classifying spaces of permutation representations because the associated covering space is not a fiber bundle (the fibers are not homeomorphic to the group). Several authors have investigated classifying spaces of permutation representations under other guises, for example, Bredon [2].

V. Joins. Let $(G, X)$ and $(L, Y)$ be two permutation representations. Define the join of $(G, X)$ and $(L, Y)$, denoted $(G, X) *(L, Y)$, to be the permutation representation $(G \times L, X \amalg Y)$, where $(g, l) x=g x$ and $(g, l) y=l y$ gives the action of $G$.

Proposition 5.1. A classifying space for $(G, X) *(L, Y)$ is given by $B_{(G, X)}$ * $B_{(L, Y)}$, where $B_{(G, X)}$ and $B_{(L, Y)}$ are classifying spaces for $(G, X)$ and $(L, Y)$, respectively.

Proof. Let $E=E_{1} * E_{2}$, where $E_{1}$ and $E_{2}$ are spaces satisfying the hypotheses of Theorem 3.2 for $(G, X)$ and $(L, Y)$, respectively. Let $G \times L$ act on $E$ by

$$
(g, l)\left(t_{0} e_{0}, t_{1} e_{1}\right)=\left(t_{0} g e_{0}, t_{1} g e_{1}\right),
$$

where $t_{0}+t_{1}=1$. Then $G \times L$ acts on the cells of $E$ by $(g, l)(\sigma * \tau)$ $=g \sigma * l \tau$. We show that $E$ is $(G, X) *(L, Y)$-contractible and that $G \times L$ acts on $E$ with the right stabilizers.

Denote the stabilizer sets of $(G, X)$ and $(L, Y)$ by $\mathfrak{\oiint}_{X}$ and $\mathfrak{S}_{Y}$, respectively. Then the stabilizer set of $(G, X) *(L, Y)$ is

$$
\mathfrak{S}_{*}=\left\{H \times L, G \times K \mid H \in \mathfrak{Q}_{X}, K \in \mathfrak{Q}_{Y}\right\}
$$

If $\sigma * \tau \in E$, then the stabilizer of $\sigma * \tau$ is $H \times K$, where $H$ fixes $\sigma$ and $K$ fixes $\tau$. We have $H \times K=(H \times L) \cap(G \times K) \in \mathfrak{G}_{*}^{\prime}$, and $H \times K \neq G \times L$ because at least one of $\sigma$ and $\tau$ is a nondegenerate cell. Thus $G \times L$ acts on $E$ with the right stabilizers.

To show $E$ is $\mathfrak{G}_{*}$-contractible, we can, without loss of generality, choose $H \in \mathfrak{S}_{X}$ and $K=G$ or $K \in \mathfrak{B}_{Y}$, and prove $E$ is $H \times K$-contractible. Let $F$ be an $H$-contracting homotopy for $E_{1}$ contracting the identity map to the constant map on $v$. Define

$$
F^{\prime}\left(\left(t_{0} e_{0}, t_{1} e_{1}\right), t\right)=\left(t_{0} F\left(e_{0}, t\right), t_{1} e_{1}\right)
$$


Then $F^{\prime}$ is equivariantly proper. By Lemma 4.1 , let $F^{\prime \prime}$ be an $H \times K$ contracting homotopy from the identity on $v * E_{2}$ to the constant map on $v$. Define $F: E \times I \rightarrow E$ by

$$
\begin{array}{ll}
F(e, t)=F^{\prime}(e, 2 t) & \text { for } 0 \leqslant t \leqslant \frac{1}{2}, \\
F(e, t)=F^{\prime \prime}\left(e, 2\left(t-\frac{1}{2}\right)\right) & \text { for } \frac{1}{2} \leqslant t \leqslant 1 .
\end{array}
$$

Then $F$ is an $H \times K$-homotopy from the identity on $E$ to the constant map on $v$, and is equivariantly proper.

By Theorem 3.2, $E /(G \times L)$ is a classifying space for $(G, X) *(L, Y)$. The following lemma will show that $E /(G \times L)=B_{(G, X)} * B_{(L, Y)}$, which is what we want:

LEMMA 5.1. If the groups $G$ and $L$ act on cell complexes $E_{1}$ and $E_{2}$ respectively, then $E_{1} / G * E_{2} / L$ is homeomorphic to $\left(E_{1} * E_{2}\right) /(G \times L)$.

Proof. Define

$$
\varphi: E_{1} / G * E_{2} / L \rightarrow\left(E_{1} * E_{2}\right) /(G \times L)
$$

and

$$
\psi:\left(E_{1} * E_{2}\right) /(G \times L) \rightarrow E_{1} / G * E_{2} / L
$$

by

$$
\begin{aligned}
\varphi\left(\left(t_{1} r\left(e_{1}\right), t_{2} r\left(e_{2}\right)\right)\right) & =r\left(\left(t_{1} e_{1}, t_{2} e_{2}\right)\right), \\
\psi\left(r\left(t_{1} e_{1}, t_{2} e_{2}\right)\right) & =\left(t_{1} r\left(e_{1}\right), t_{2} r\left(e_{2}\right)\right),
\end{aligned}
$$

where $r$ is used to denote quotient maps. It is trivial to check that $\varphi$ and $\psi$ are well-defined maps and that $\varphi=\psi^{-1}$. The lemma now follows from the fact that the map $E_{1} \times I \times E_{2} \rightarrow E_{1} / G \times I \times E_{2} / L$ is an identification map.

Let $(G, X)$ be a permutation representation, and let $E$ be a classifying space for it. Let $\beta: H^{*}(E / G, Z) \rightarrow H^{*}((G, X), Z)$ be the isomorphism of Theorem 3.2. Then we have the following:

Proposition 5.2. Let $(G, X)$ be a permutation representation with stabilizer set

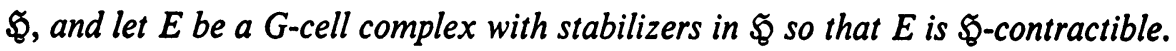
Then

$$
\beta(u \cup v)=\beta u \cup \beta v
$$

for all $u, v \in H^{*}(E / G, Z)$.

Proof. The cup product is defined from the map $\delta: E \rightarrow E \times E$ given by $\delta(e)=(e, e)$. The map $\delta$ is not cellular but is carried by the acyclic carrier 
$C(\sigma)=\mathrm{Cl}(\sigma) \times \mathrm{Cl}(\sigma)$. This carrier yields a $G$-chain map $\delta^{*}: C_{*}(E) \rightarrow C_{*}(E)$ $\otimes C_{*}(E)$, unique up to $C$-carried $G$-homotopy by Corllary 3.1. Then $\delta^{*}$ satisfies the conditions for a diagonal map in the cohomology theory of permutation representations, and we can use it to define the product on $H^{*}((G, X), Z)$. The space $E / G$ likewise has a diagonal map $d$, which is induced by $\delta$, so that $d(e)=(e, e)$. This map is carried by the acyclic carrier $D(\sigma)=\mathrm{Cl}(\sigma) \times \mathrm{Cl}(\sigma)$, where $D$ is induced by $C$. The corresponding map for $C_{*}(E / G)$ turns out to be

$$
\begin{aligned}
i \circ\left(\delta^{*} \otimes 1\right): C_{*}(E) \otimes_{G} Z & \rightarrow\left(C_{*}(E) \otimes C_{*}(E)\right) \otimes_{G \times G} Z \\
& \cong\left(C_{*}(E) \otimes_{G} Z\right) \otimes\left(C_{*}(E) \otimes_{G} Z\right) .
\end{aligned}
$$

The proposition now follows routinely from manipulations as in the proof of Theorem 3.2.

The following theorem appeared in Blowers [1], where a lengthy algebraic proof was referred to but not shown. A brief topological proof will now be presented.

THEOREM 5.1. Let $(G, X)$ and $(L, Y)$ be permutation representations. Then the cup product on $H^{*}((G, X) *(L, Y), Z)$ is trivial; i.e., if

$$
\alpha \in H^{n}((G, X) *(L, Y), Z) \text { and } \beta \in H^{p}((G, X) *(L, Y), Z),
$$

with $n, p>0$, then $\alpha \beta=0$.

Proof. Let $B_{(G, X)}$ and $B_{(L, Y)}$ be classifying spaces for $(G, X)$ and $(L, Y)$, respectively. By Proposition 5.1, $B_{(G, X)} * B_{(L, Y)}$ is a classifying space for $(G, X) *(L, Y)$. Further, it is the join of two spaces. This is homotopically equivalent to the suspension of their smash product (Brown [3, p. 168]), and the product on any suspension is trivial (Steenrod and Epstein [10, p. 4]). By Proposition 5.2, the product on $H^{*}((G, X) *(L, Y), Z)$ is trivial in positive dimensions.

\section{BIBLIOGRAPHY}

1. James V. Blowers, The cohomology rings of certain finite permutation representations, Proc. Amer. Math. Soc. 45 (1974), 157-163.

2. Glen Bredon, Equivariant cohomology theories, Lecture Notes in Mathematics, No. 34, Springer-Verlag, Berlin and New York, 1967. MR 35 \#4914.

3. Ronald Brown, Elements of modern topology, McGraw-Hill, New York, 1968. MR 37 \#3563.

4. George E. Cooke and Ross L. Finney, Homology of cell complexes, Princeton Univ. Press, Princeton, N.J.; Univ. of Tokyo, Tokyo, 1967. MR 36 \#2142.

5. Samuel Eilenberg and J. C. Moore, Foundations of relative homological algebra, Mem. Amer. Math. Soc. No. 55 (1965). MR 31 \#2294.

6. Morton E. Harris, Generalized group cohomology, Fund. Math. 65 (1969), 269-288. MR 40 \#5701. 
7. G. Hochschild, Relative homological algebra, Trans. Amer. Math. Soc. 82 (1956), 246-269. MR 18, 278.

8. John Milnor, Construction of universal bundles. II, Ann. of Math. (2) 63 (1956), 430-436. MR $17,1120$.

9. Ernst Snapper, Cohomology of permutation representations. I. Spectral sequences, J. Math. Mech. 13 (1964), 133-161. MR 28 \#1226.

10. Norman E. Steenrod and D. B. A. Epstein, Cohomology operations, Princeton Univ. Press, Princeton, N.J., 1962. MR 26 \#3056.

Current address: 6 Canterbury Drive, Chambersburg, Pennsylvania 17201 